\title{
Commentary of the Special Panel at the Hopkinton Conference Working Group on Workplace Disability Prevention
}

\author{
Robert K. McLellan' ${ }^{1}$ Paula Aznavoorian-Barry ${ }^{2}$ David Brown ${ }^{3}$. \\ Edmund C. Corcoran $\mathrm{Jr}^{4} \cdot$ Garry Pearce ${ }^{5}$
}

Published online: 3 October 2016

(C) Springer Science+Business Media New York 2016

Medicine has long prided itself on the use of evidence to inform practice. For centuries, physicians followed Hippocrates lead by closely observing individual patients to recognize patterns characteristic of certain diseases. Doctors considered responses to treatment of their last patient to plan therapeutics for the next patient with a similar syndrome. The doctor was intimately involved in "research," which was then immediately incorporated into his or her practice. However, scientific contributions over the past few decades have led to the recognition that anecdotal approaches to planning treatment resulted in wide variation in practice and outcomes. The term "evidence-based medicine" came of age about 25 years ago and led to the creation of rigorous methodologies to identify effective therapies that would benefit most patients. The results of successful clinical studies were then codified in practice guidelines, but the translation of evidence into practice has not gone well. As the scientific method evolved to the current gold standard of randomized controlled trials, the distance between those who gather evidence and those who are expected to apply it broadened to a chasm. The science

Robert K. McLellan

Robert.K.McLellan@dartmouth.edu

1 Dartmouth-Hitchcock Medical Center, One Medical Center Dr., Lebanon, NH 03756, USA

2 Liberty Mutual Insurance, 100 Liberty Way, Dover, NH 03820, USA

3 Canadian Imperial Bank of Commerce, 333 Bay Street, Suite 1500, Toronto, ON M5H 2R2, Canada

4 Raytheon Company, Global Business Services, 870 Winter Street, Waltham, MA 02451, USA

5 University of Sydney, 5 Moseley St, Carlingford, Sydney, NSW 2118, Australia and practice of disability management serve as an exemplar of this problem. Refreshingly, the "Hopkinton Conference Working Group on Disability Prevention and Management" convened a special panel of end users of disability research to take part in the deliberations of the investigators. This brief commentary reflects on our experience with the challenges of applying evidence and how we think our presence at this conference may not only help facilitate the translation of research to practice but also how practice should and can advise research.

Change agents in virtually every field have learned a hard lesson: delivering a new process to a group uninvolved in its development with the expectation of universal adoption does not work and may end up with unintended adverse consequences. Participatory research has evolved in response to this problem in a number of fields ranging from creating the next pop star or fad product to solving community health problems. Investigators have recognized a broad spectrum of stakeholders with differing values and priorities involved in disability prevention and management. Implementation of a new, evidence-based practice often requires changed behavior of many people and calls for an entire system to work together to succeed. The numerous participants in return-to-work processes form a web of influence, with any one individual having the power to sabotage an evidence-based practice. By engaging stakeholders in the process of research design, barriers to implementation can be identified and mitigated.

The inclusion of disability practitioners in research also makes sense because they may have developed effective methods that deserve investigation. The science of quality improvement effectively uses learning collaboratives of practitioners to identify best of class outcomes. Distinguishing practices of the successful teams are then disseminated for trial in other settings. 
In the medical world, shared decision-making has emerged as a tool to assure that health providers accord the patient's priorities the highest respect. Such an instrument is lacking in workability management. One reason it may be lacking is that the employee patient's values may conflict with those of the employer or other more powerful stakeholders. In practice, the art and science of negotiation and conflict resolution power results. Shouldn't these social sciences play a significant role in future disability research?

The scientific challenge of both conducting and applying field research is that it is messy. Uncontrolled secular events may overcome the power of an intervention. Employers have very different cultures, priorities, ways of doing things, and are on a spectrum of readiness to change their systems. We experience this diversity and challenge in spades. Disability interventions are likely to be effective in different organizations depending on their willingness to change. Medical missionaries have learned that understanding the health beliefs of a population is foundational to any effective therapeutic. So too, as managers of disability, we know that understanding our local culture is critical to applying evidence about disability management. We must use our anthropological skills to apply effectively published research to our practice settings.

Disability publications usually lack sufficient details about the step by step methods used to launch, operate and sustain an effective practice. More attention should be devoted to translation science that presents evidence to consumers along with easy to use tools to address multiple users' roles and needs. It is one thing to know that washing hands prevents transmission of contagious diseases. It is another thing altogether to construct a highly reliable system to assure that health care workers consistently wash their hands. Systems are highly resistant to change. A research project employs external means to conduct an experiment, but these resources usually disappear once the study has concluded. The application of disability science to consistent practice requires attention to the factors necessary for sustainability, or the system will soon revert to status quo.

The complexity of disability management is rapidly evolving with the transformation of workforce demographics and the nature of work, its organization and its place. With these changes, traditional methods to maintain and promote workability are increasingly outdated. Practitioners are left without much evidence to apply to this new world.

The siloed nature of disability benefits, statutes, and regulations as they relate to work versus non-work related disability has helped perpetuate a system that ignores the interaction between "work" and "non-work related" disability. As well, employers frequently do not extend stay- at-work/return-to-work programs to short-term disability. Too often, the federal safety net of social security becomes an easy out for ineffective private disability interventions, shifting the cost to a system that cannot afford it. We believe it is time to bring together participants in the private and public sector to address the need to develop an integrated approach across the spectrum of causation of disability that recognizes the roles that employee, employer, community and society as a whole can play in preserving human workability.

Although disability benefits burden businesses and society, health economists have pointed out that unscheduled absence and presenteeism likely exceeds standard disability costs because of unexpected disruptions of daily business operations. Unlike unscheduled absence, disability absence is predictable. An employer can add staff, reschedule operations and otherwise mitigate expected absence. Effective approaches to absence management need to be better investigated. Further, although alternate duty is a proven approach to reducing the hard dollar costs associated with disability, research should better account for the potential impact of employees at work who are not "100\%." There may be a rational reason why many employers have historically resisted alternative duty programs.

Recognizing the role that employee health and wellbeing plays in disability, absence, and presenteeism, the majority of employers have adopted strategies to promote the health of their employees. Some have extended their reach to provide more support for chronic disease management. The most progressive companies have adopted an overarching framework for managing the health and health-related productivity of their workforce called Total Worker Health ${ }^{\circledR}$ by The National Institute for Occupational Safety and Health. This approach, founded on the demonstrated inextricable relationship between health and work, incorporates all "policies, programs, and practices that integrate protection from work-related safety and health hazards with promotion of injury and illness prevention efforts to advance worker well-being [1]." We believe that the emerging field of Total Worker Health is poised to advance research relevant to preserving and promoting workability.

In sum, as consumers of disability research, we wholeheartedly applaud the organizers of this workshop to include us as stakeholders to inform their work. We see our influence throughout the papers. It was helpful to us as well to recognize the challenges of disability science. With this commentary, we hope to extend our influence to others in the field. From our perspective, there is a great need for interdisciplinary research that involves not only the variety of scientific disciplines relevant to disability but also the spectrum of stakeholders' views. 


\section{Compliance with Ethical Standards}

Conflict of interest Robert McLellan declares that Liberty Mutual Research Institute has paid travel expenses to a national disability conference to present his perspective and programs related to the prevention and management of disability. He collaborates with scientists at the Liberty Mutual Research Institute in research projects. Staff working for him have been reimbursed for time devoted to this research. He was provided lodging for participation as a member of the Special Panel in the Hopkinton Conference. Paula AznavoorianBarry is an employee of Liberty Mutual Insurance, which owns and operates the Liberty Mutual Research Institute which sponsored the Hopkinton Conference Working Group on Workplace Disability Prevention and sponsors disability research. David Brown was provided lodging for participation as a member of the Special Panel in the Hopkinton Conference. Edmund C. Corcoran Jr declares that he has no conflict of interest. Garry Pearce was provided lodging for participation as a member of the Special Panel in the Hopkinton Conference.

Ethical Approval This article does not contain any studies with animal or human participants performed by any of the authors.

\section{Reference}

1. NIOSH, National Occupational Research Agenda (NORA): National Total Worker Health Agenda (2016-2026): A National Agenda to Advance Total Worker Health Research, Practice, Policy, and Capacity. U.S. Department of Health and Human Services (DHHS), Centers for Disease Control and Prevention, and NIOSH. 2016. DHHS (NIOSH) publication 2016-119. http://www. cdc.gov/niosh/docs/2016-114/pdfs/nationaltwhagenda2016-1144-14-16. pdf. Accessed 21 Aug 2016. 